\title{
Fully Plastic J-Integrals for Mixed Mode Fracture Induced by Inclined Surface Cracks in Pressurized Ductile Pipes
}

\author{
Weigang Wang ${ }^{1}$, Wei Yang ${ }^{2}$, and Chun-Qing $\mathrm{Li}^{1}$ \\ ${ }^{1}$ RMIT University \\ ${ }^{2}$ The University of Melbourne
}

November 24, 2020

\begin{abstract}
Surface cracks have been recognized as major causes for fracture failures of ductile pipes. This paper intends to derive a normalized fully plastic J-integral solution to mixed-mode fracture caused by inclined surface cracks in pressurized ductile pipes. A combined J-integral and finite element method is developed to evaluate the J-integral for inclined surface cracks. A set of predictive formulas for normalized fully plastic J-integrals are developed. It is found in this paper that the normalized fully plastic J-integral increases with the decrease of crack inclination angle and aspect ratios, and the increase of strain hardening exponent. It is also found that the critical locations of crack propagation occur between the surface point and the deepest point of cracks when the inclination angle is relatively small. The paper concludes that the developed formulas can accurately predict the normalized fully plastic J-integrals along the front of inclined surface cracks. The results presented in the paper can enable researchers and practitioners to accurately predict the mixed-mode fracture failure of pressurized pipes subject to inclined surface cracks.
\end{abstract}

\section{Hosted file}

WG08_fully plastic J_to submit_231120.pdf available at https://authorea.com/users/333336/ articles/494847-fully-plastic-j-integrals-for-mixed-mode-fracture-induced-by-inclinedsurface-cracks-in-pressurized-ductile-pipes 